\title{
Measuring Beta Function and Phase Advance in RḦHC with an AC Dipole *
}

\author{
M. Bai, S. Peggs, T. Roser, T. Satogata, D. Trbojevic \\ Brookhaven National Laboratory, Upton, NY 11973, USA
}

\section{Abstract}

High energy hadron collider operation requires accurate measurements of the beta functions and phase advances, to check the linear optics and to locate gradient errors. During the RHIC 2003 run, two AC dipoles with vertical and horizontal magnetic field [1] were used to measure the linear optics at storage and at injection energies. The two $\mathrm{AC}$ dipoles are set up to adiabatically induce sizable coherent oscillations at a frequency close to the betatron frequencies. The beta functions and phase advances are then calculated from the 1024 turn-by-turn measurements available from all the RHIC BPMs (Beam Position Monitors). Because the coherent excitation is adiabatic, the beam emittance is preserved after the measurement. The algorithm is discussed in this paper, and experimental results are presented.

\section{INTRODUCTION}

During both the RHIC FY 2003 deuteron-Au run and polarized proton run, $\beta^{*}=1 \mathrm{~m}$ optics were used to maximize the luminosity. A set of beta function measurements were carried out by using the two RHIC AC dipoles to check the linear optics.

In linear accelerator, an AC dipole can be used to adiabatically generate a sustained transverse coherent oscillation without sacrificing the beam emittance [2]. The driven coherent oscillation at location $s$ is given by

$$
z(s)(i)=\frac{B_{m} L}{4 \pi B \rho \delta} \sqrt{\beta(s) \beta_{0}} \sin \left(2 \pi \nu_{m} i+\phi(s)\right)
$$

where $\left.z_{(} s\right)$ is the transverse coherent oscillation amplitude at location $s, B \rho$ is the magnetic rigidity, $B_{m} L$ is the amplitude of the integrated AC dipole oscillating field strength, $\phi(s)$ is the phase at location $s, \beta(s)$ is the betatron function at location $s, i$ is the turn number, and $\beta_{0}$ is the betatron function at the AC dipole. $\delta$ is the separation between the intrinsic betatron tune $\nu_{z}$ and the AC dipole tune $\nu_{m}$,

$$
\nu_{m} \equiv \frac{f_{m}}{f_{r e v}}
$$

where $f_{m}$ is the AC dipole oscillation frequency and $f_{r e v}$ is the beam revolution frequency. Hence, the ratio of the square root beta functions at two different locations is equal

* The work was performed under the auspices of the US Department of Energy to the ratio of the coherence amplitudes at these two locations. The phase difference between the two locations is the true phase advance only if the AC dipole is not in-between.

In general, the transverse oscillation at two different locations $s_{1}$ and $s_{2}$ is given by

$$
\left(\begin{array}{c}
z_{2} \\
z_{2}^{\prime}
\end{array}\right)=M\left(\begin{array}{c}
z_{1} \\
z_{1}^{\prime}
\end{array}\right)
$$

where $z_{1,2}$ is the transverse position of the beam, $z_{1,2}^{\prime}$ is the transverse angle of the trajectory. $M$ is the transfer matrix between the two locations and

$$
\begin{gathered}
M=\left(\begin{array}{ll}
m_{11} & m_{12} \\
m_{21} & m_{22}
\end{array}\right)= \\
\left(\begin{array}{cc}
\sqrt{\frac{\beta_{2}}{\beta_{1}}}\left(C+\alpha_{1} S\right) & \sqrt{\beta_{1} \beta_{2}} S \\
\frac{\alpha_{1}-\alpha_{2}}{\sqrt{\beta_{1} \beta_{2}}} C-\frac{1+\alpha_{1} \alpha_{2}}{\sqrt{\beta_{1} \beta_{2}}} S & \sqrt{\frac{\beta_{1}}{\beta_{2}}}\left(C-\alpha_{2} S\right)
\end{array}\right)
\end{gathered}
$$

where $S=\sin \phi_{12}, C=\cos \phi_{12}, \beta_{1,2}$ are the beta functions at location $s_{1}, s_{2}, \alpha_{1,2}=-\frac{\beta_{1,2}^{\prime}}{2}$ and $\phi_{12}$ is the phase advance between the two locations and [3]

$$
\frac{1}{\beta_{1}}\left(\frac{C}{S}+\alpha_{1}\right)=\frac{m_{11}}{m_{12}}
$$

Assuming that the turn by turn coherent oscillation is measured at three beam position monitors 1,2 and 3 with phase advances in between of $\phi_{12}$ and $\phi_{23}$, the beta function at BPM 1 is given by

$$
\beta_{1}=\beta_{1}^{m} \sqrt{\frac{\beta_{2} / \beta_{1}}{\beta_{2}^{m} / \beta_{1}^{m}}} \frac{\sin \phi_{12}^{m}}{\sin \phi_{12}}
$$

where $\beta_{1}$ is the measured beta function at BPM $1, \beta_{1}^{m}$ is the model beta function at BPM $1, \phi_{i j}$ is the measured phase advance between BPM $\mathrm{i}$ and BPM $\mathrm{j}$ where $i, j=1,2,3$, and $\phi_{i j}^{m}$ is the model phase advance between BPM $i$ and $B P M j$.

\section{RHIC LINEAR OPTICS MEASUREMENT}

In RHIC, transverse coherent betatron oscillations were adiabatically excited by the horizontal (or vertical) AC dipole. The amplitude of the AC dipole magnetic field was ramped up in 10,000 turns. It was then kept at constant for 2,000 turns for one to take the turn-by-turn beam position data and then ramped down to zero in another 10,000 turns. Since the coherent oscillation was adiabatically excited, the frequency spectrum of this driven oscillation is 

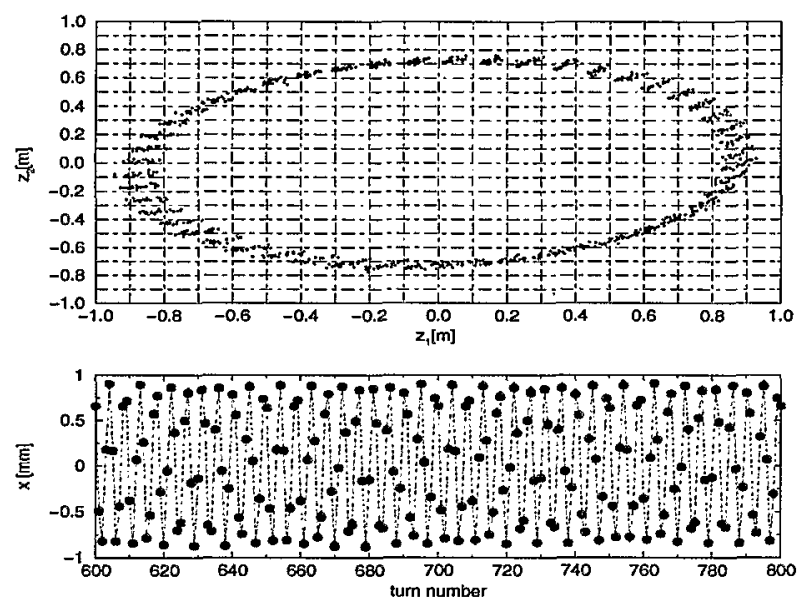

Figure 1: The bottom figure shows the vertical turn-byturn beam position data in the middle of the arc. The black solid circles are the measured turn-by-turn beam position data and the open diamonds are the fitted turn-by-turn data. The phase plot at the top is the 1024 turn data at the BPM and the BPM next to it.

dominated by the frequency of the AC dipole. Thus, the coherent oscillation amplitude $z_{m}$ and phase $\phi$ can be obtained by fitting the turn by turn beam position data by

$$
z(i)=z_{m 1} \cos \left(\nu_{m} 2 \pi i\right)+z_{m 2} \sin \left(\nu_{m} 2 \pi i\right)
$$

where $z(i)$ is the horizontal(or vertical) position of $i^{t h}$ revolution turn. The amplitude $z_{m}$ and phase $\phi$ of the coherent oscillation are given by

$$
z_{m}=\sqrt{z_{m 1}^{2}+z_{m 2}^{2}}
$$

and

$$
\phi=\arctan \left(\frac{z_{m 2}}{z_{m 1}}\right) .
$$

Fig. 1 shows the typical turn-by-turn vertical coherent oscillation at a BPM located in the arc area, and the phase plot represented by using the 1024-turn BPM data of two adjacent BPMs. The spread of the data points in the phase plot is about $0.1 \mathrm{~mm}$ and is about same level of the noise on the BPM turn-by-turn signal.

In RHIC, there is a total of 492 physical BPMs distributed around each ring. Among them, 84 are dual-plane BPMs. The vertical BPMs are located at every defocusing quadrupoles, and the horizontal BPMs are located at focusing quadrupoles [4]. 1024 turns of beam position at each BPM were collected with the AC dipole oscillating at a fixed frequency and fix amplitude. The amplitude and phase of the coherent oscillation driven by the AC dipoles at each beam were extracted using Eq. 8 and Eq. 9 . The phase difference between any two BPMs is the phase advance in between, and the beta function at each BPM around the ring can be obtained by using Eq. 6 . Fig. 2 is a typical example of the measured beta functions and phase advance at storage energy in the RHIC Yellow ring with $1 \mathrm{~m} \beta^{*}$ at IP 8 and $2 \mathrm{~m} \beta^{*}$ at IP 6, IP 10 and IP 2 . Compared with the model optics, the rms deviation of the measured phase advances and beta functions from the predicted value is about $12 \%$ in the arc area and larger in the interaction area.

In order to provide enough aperture around the RHIC beam abort area at injection, a lattice with $\beta^{*}=10 \mathrm{~m}$ at all IPs was used at injection. At storage energy, a lattice of $2 \mathrm{~m}$ $\beta^{*}$ at IP 6, IP 10 and IP 2 and $1 \mathrm{~m} \beta^{*}$ at IP 8 was used [5]. In RHIC, three quadrupoles (triplet) were placed next to the $D 0$ magnet in the interaction region for achieving the desired small $\beta^{*}$ (beta function at interaction point) [6]. Normally, the beta function at the triplets grows considerably when the $\beta^{*}$ gets squeezed down and any gradient error in the triplet quadrupoles then gets amplified and can result in beta function waves through the whole lattice. Fig 3 shows the measured beta function wave with the two different lattices. The larger beta wave of the $\beta^{*}=1 \mathrm{~m}$ lattice than the one of the $\beta^{*}=10 \mathrm{~m}$ lattice indicates the effect of the triplets effect.

\section{CONCLUSION}

The horizontal and vertical AC dipole magnets were commissioned during the RHIC FY 2003 run to measure the beta functions and phase advances at injection as well as at store. Several measurements were done in both Blue ring and Yellow ring to confirm the beta functions at the RHIC interaction point of lattices with different $\beta^{*}$ lattices.

\section{ACKNOWLEDGEMENT}

The authors would like to thank Dr. Johannes van Zeijts for the fruitful discussions. The authors would also like to thank L. Hoff, V. Ponnaiyan, J. Piacentino and B. Oerter on the constructing the control hardware and software. The authors would also like to thank P. Oddo, J. Delong, T. Russo, D. Lehn, T. Curcio and other engineers for their help.

\section{REFERENCES}

[1] M. Bai, et al, RHIC AC Dipole Design and Construction, Proceedings of the 2001 Particle Accelerator Conference, p. 3606 (June 2001).

[2] M. Bai et al., Physical Review E, 5 (1997).

[3] Castro-Garcia P., Luminosity and Beta Function Measurement at the Electron-Positron Collider Ring, LEP: Doctoral Thesis, Geneva, 1996.

[4] T. Satogata, et al, RHIC Beam Instrumentation, Nuclear Instruments and Methods in Physics Research A 499 (2003) 372-387

[5] T. Satogata et al., Linear Optics Measurement and Correction in the RHIC 2003 Run "Optics, Proceedings of the 2003 Particle Accelerator Conference(May 2003).

[6] RHIC Design Manual, April 1998. 


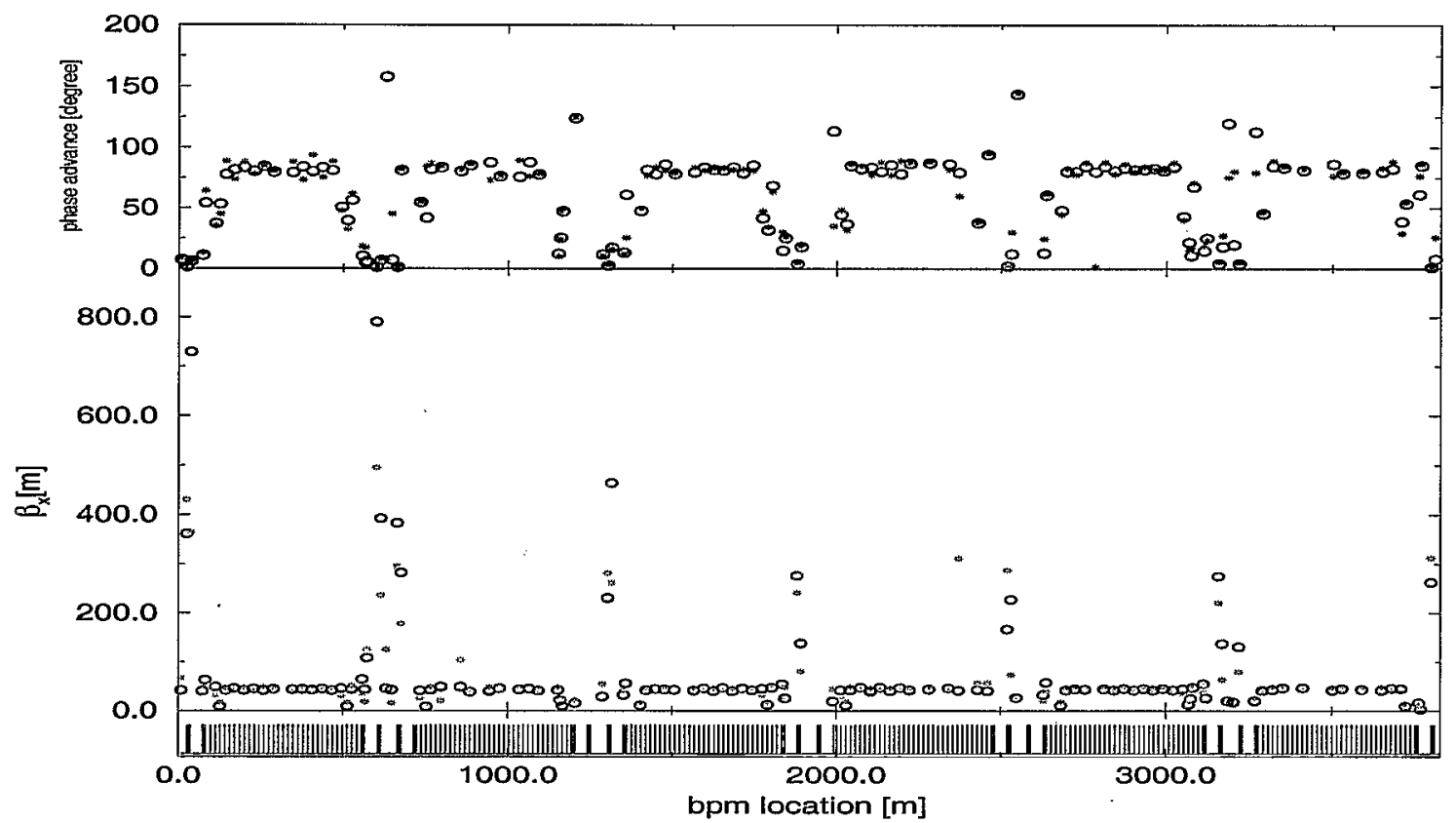

Figure 2: The bottom plot is the schematic layout of dipoles in the RHIC lattice showing 6 IPs. The stars in the middle plot are the horizontal betatron functions at all the available yellow BPMs measured at the RHIC Au storage using the vertical AC dipole. The open circles represent the horizontal beta functions predicted by the RHIC model. In the plot at the top, the stars are the horizontal phase advances between every two adjacent yellow BPMs measured at the Au storage energy. The open circle represents the corresponding phase advances predicted by the RHIC model. Because of the missing BPMs, the algorithm assigns the predicted phase advance to a BPM if the one next to it is missing.

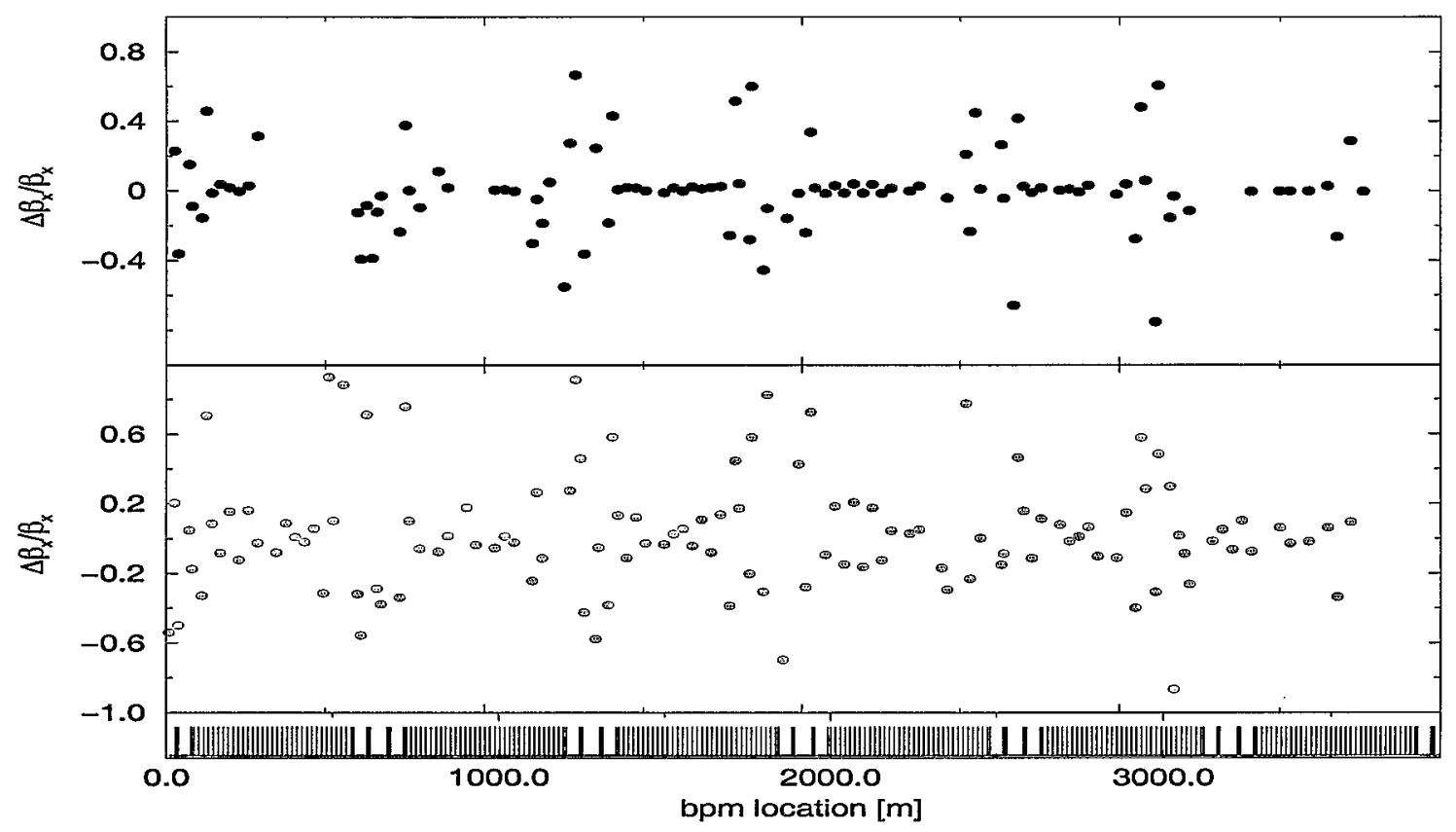

Figure 3: The bottom plot is the schematic layout of dipoles in the RHIC lattice. The stars in the middle plot and top plot are the differences of the measured horizontal beta functions and the beta functions in the model normalized of the predicted beta functions at all the available yellow BPMs measured at RHIC Au storage energy and injection energy. 